Document downloaded from:

http://hdl.handle.net/10251/78063

This paper must be cited as:

Navarro -Martinez, IJ.; Villalba Sanchis, I.; Martínez Fernández, P.; Insa Franco, R. (2015). Analytical model for predicting the buckling load of continuous welded rail tracks.

Proceedings of the Institution of Mechanical Engineers, Part F: Journal of Rail and Rapid Transit. 229(5):542-552. doi:10.1177/0954409713518039.

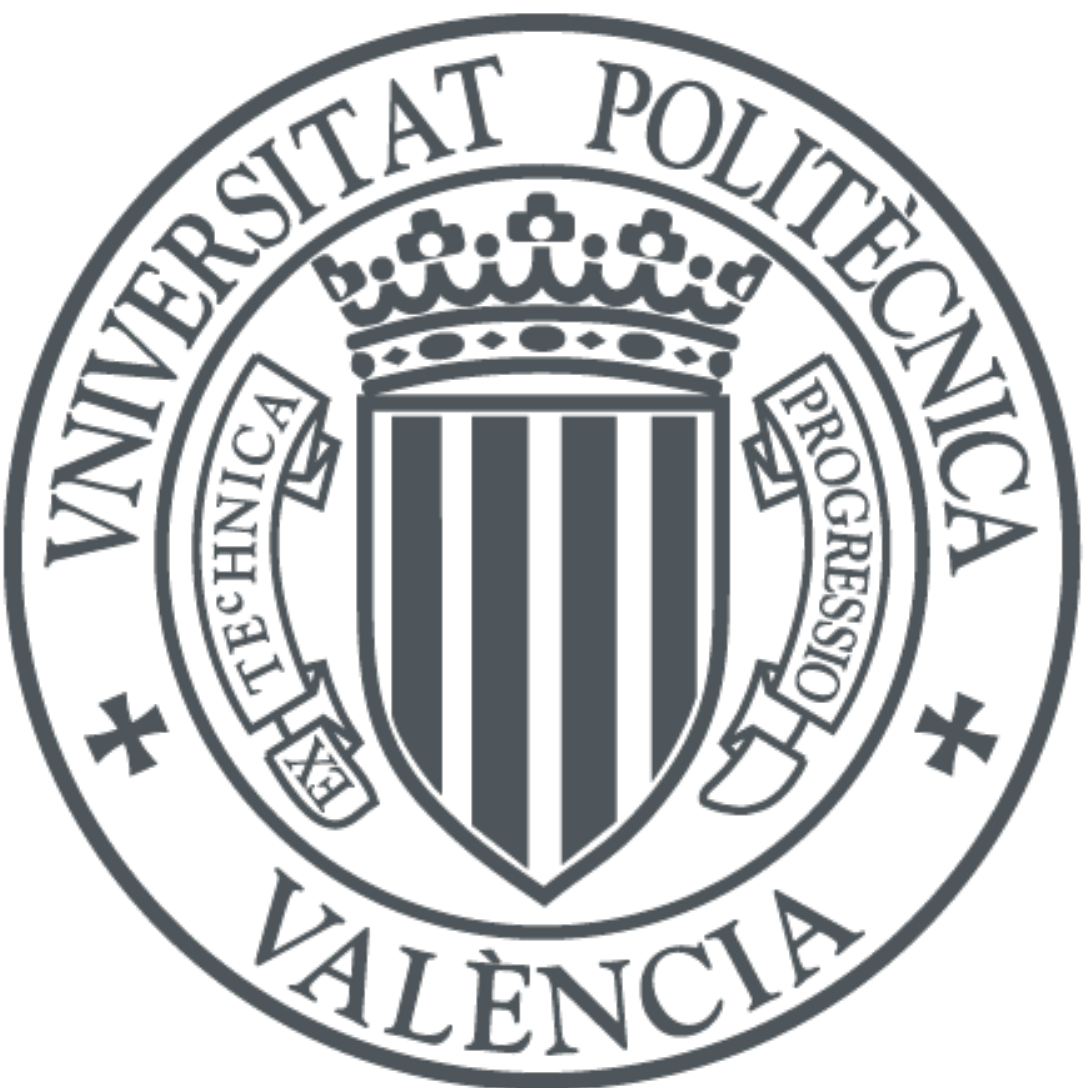

The final publication is available at

http://dx.doi.org/10.1177/0954409713518039

Copyright SAGE Publications (UK and US)

Additional Information 


\title{
Analytical Model for Predicting the Buckling Load of Continuous Welded Rail
}

\section{Tracks}

Authors: Ignacio Navarro Martínez, Ignacio Villalba Sanchis, Pablo Martínez Fernández, Ricardo Insa Franco

Affiliation: Departamento de Ingeniería e Infraestructura de los Transportes. Universidad Politécnica de Valencia, Camino de Vera, 46022 Valencia (Spain)

Corresponding Author: Ignacio Navarro Martínez. Tel.:+34675265764. E-mail address: ignamar1@cam.upv.es

\begin{abstract}
Continuous Welded Rail (CWR) track solved many of the problems associated to tread surface discontinuities of the jointed tracks. However, as longitudinal expansion of the rails in CWR tracks is highly constrained, compressive stresses in rails may cause track buckling in the horizontal plane. Track buckling is a complex phenomenon, in which many factors are involved and around which there is much uncertainty. The objective of this paper is to present an analytical model with which to calculate the buckling load of a CWR track. This model accounts for different contribution of base, crib and shoulder ballast and includes the affection of vertical loading to each of these components. Moreover, a parametrical study based on this model was developed, in order to understand how and how much the considered factors affect the track stability. The results of the survey indicated that the characteristics of the existing misalignments in the track were the most critical parameters involved in the phenomenon. Moreover, the maintenance operations that affect ballast, such as tamping or surfacing, and the dimensions and material of the track sleepers proved to be very important as well.
\end{abstract}




\section{Keywords}

Buckling, Continuous Welded Rail, Parametric Study, Energy Method, Ballast Resistance.

\begin{tabular}{|c|c|}
\hline Nomencl & \\
\hline$V$ & System's total potential \\
\hline$U$ ballast & Energy associated to ballast resistance \\
\hline$U$ bending & Deformation energy associated to rail bending \\
\hline$W_{\mathrm{p}}$ & Work of the external forces \\
\hline$q_{\mathrm{i}}$ & Generalized coordinate $i$ \\
\hline$P$ & Applied external force \\
\hline$E I_{\mathrm{h}}$ & Rail bending rigidity in the horizontal plane \\
\hline$L$ & Length of the misalignment \\
\hline$f_{\mathrm{o}}$ & Amplitude of the initial misalignment \\
\hline$y_{\mathrm{o}}$ & Amplitude of the track lateral deflection \\
\hline$\varphi$ & Ballast total resistance \\
\hline$\varphi_{\mathrm{b}}$ & Base component of ballast resistance \\
\hline$\varphi_{\mathrm{b}}{ }^{*}$ & Amplified base component of ballast resistance \\
\hline$\varphi_{\mathrm{e}}$ & Sleeper end component of ballast resistance \\
\hline$\varphi_{\mathrm{c}}$ & Crib component of ballast resistance \\
\hline$N_{\mathrm{x}}$ & Vertical load per unit of length \\
\hline$Q$ & Vertical axle load \\
\hline$N_{\mathrm{x}}^{\mathrm{Q}}$ & Traffic vertical load per unit of length \\
\hline$\mu$ & Friction coefficient between sleeper and ballast \\
\hline$\delta$ & Interface friction angle for the sleeper base \\
\hline$K_{\mathrm{p}}$ & Passive earth pressure coefficient \\
\hline$K_{\mathrm{o}}$ & Coefficient of earth pressure at rest \\
\hline
\end{tabular}




\begin{tabular}{|ll|}
\hline $\begin{array}{l}N \\
\gamma^{\prime}\end{array}$ & Resultant of the earth pressure at rest \\
$a$ & Cocific weight of ballast \\
$h$ & Sleeper length \\
$b$ & Sleeper height \\
$y$ & Sleeper width \\
$x$ & Track lateral deflection \\
$X_{\mathrm{G}, \mathrm{I}}$ & Track longitudinal coordinate \\
$\bar{P}_{i, j}$ & Global effect of the parameter $i$ \\
$I_{\mathrm{i}}$ & Mean buckling load for the level $j$ of the parameter $i$ \\
\hline
\end{tabular}

\section{Introduction}

In conventional railway tracks, rails are joined together mechanically by their ends, so that structural continuity is ensured and thermal expansion is allowed. These joints, however, generate a series of problems which affect negatively the passenger comfort, reduce the life time of track components and increase the maintenance costs.

During the 1930s, the Continuous Welded Rail (CWR) track was introduced in order to solve these disadvantages [1]. This track offers a continuous running surface for passing vehicles over several hundreds of meters, so that the drawbacks associated to conventional tracks disappear. However, the axial compressive stresses in the rails caused by temperature increase may lead to the buckling of the track, which is the major problem in CWR tracks. Buckling causes yearly hundreds of derailments and generates very high economic costs due mainly to reparations and the affection to the track operation [2]. 
The track instability phenomenon, in which many different factors are involved, is very complex, and nowadays there is a great uncertainty about how and how much these factors influence it. Usually, the parametric studies carried out to characterize the effect of these factors are based on finite element models [3, 4]. Because of the complexity of the proposed models, these studies frequently focus on few factors, such as the amplitude of the misalignment or the value of the resistance offered by the ballast against the lateral displacement of the buckling track.

Analytical models are also developed to study CWR track buckling. In 1978, Kerr [5] presented a beam model and applied the principle of virtual displacements so as to calculate the buckling load of the track. However, this model has several limitations. It does not consider the effect of the maintenance operations on the ballast resistance and requires a complex iterative method to solve the proposed equations. The effect of vertical loads on the lateral resistance is neither taken into account. In 2006, Grissom and Kerr [6] improved this model by considering the effect of the fasteners on the global rigidity of the track. In 1996, Samavedam [7] published a method based on the equilibrium formulation in the deformed geometry with which the lateral deflection due to a certain compressive strength could be calculated. The major limitation of this model is that it is only solved for small displacements, so that the lateral resistance offered by ballast is proportional to the displacement of the track. However, for greater lateral displacements, such as those existing in a buckled track, this hypothesis is no longer valid [8]. This model also requires an iterative method for solving the equations. In 2001, Esveld [9] presented a beam model which allowed a direct calculation of the buckling load of a CWR track. However, for simplicity, the ballast resistance was held constant and no vertical loads were considered. In 2006, López Pita [10] presented a model based on the formulation of the equilibrium in the deformed position of the track. 
This model allowed the analytical consideration of many different factors, such as the track bending rigidity exerted by fasteners or a non linear behavior of ballast. However, for the resolution of the differential equation many simplifications were done. So, this model did not handle with initial misalignments of the track, and did only consider a linear behavior for the lateral resistance offered by ballast, no matter the magnitude of the track lateral displacement.

In the model presented in this paper, some important factors involved in the buckling process are considered explicitly, such as the sleeper type, the passing of running vehicles, the maintenance operations, the form of the misalignment or the variation of the ballast height over the base of the sleepers. These factors were not considered in the previously described models. For this reason, an analytical model has been developed to study the influence of these factors on the track buckling process.

\section{Methodology}

Here, the developed analytical model is presented. The main points of the explanation refer to the consideration of ballast and its effect on the buckling load. Special attention is paid to vertical loading and variation of sleeper ballast coverage and its effect on total ballast lateral resistance.

\subsection{Theoretical background}

The buckling load of a compressed structure is the one for which the structure finds itself in a neutral equilibrium state. Taking this into account, the value of the buckling load can be obtained from the system's total potential $V$, which is a function of the generalized coordinates $q_{\mathrm{i}}$ of the structural system and of the external forces $P$. Applying the principle of stationary potential energy, the equilibrium equations can be written as 
$\frac{\partial V}{\partial q_{i}}=0 \quad(i=1,2, \ldots, n)$

In the theory of stability, a structure subjected to conservative loads reaches a neutral equilibrium state when the Hessian matrix is singular [11]. The buckling load is the one for which these conditions are fulfilled.

\subsection{Model Description}

The rail-sleeper structure is replaced by an equivalent beam, with the same cross section and inertia as the real track. The influence of the fasteners rigidity has not explicitly been taken into account in the model. Instead of that, the extra track rigidity exerted by the fasteners due to their torsion resistance is considered by applying a coefficient $\beta$ to the lateral rigidity $E I_{\mathrm{h}}$ of a single rail [12]. The value of this coefficient depends on the type of sleeper and the maintenance conditions of the fasteners, but is usually considered as $2[5]$.

A straight track with an initial sinusoidal misalignment with a length $\mathrm{L}$ and amplitude $f_{\mathrm{o}}$ has been supposed:

$f(x)=f_{0} \sin \left(\frac{n \pi x}{L}\right)$

The parameter $n$ has been used to consider different forms of the initial misalignment in the analysis, namely a half wave $(n=1)$ or a full wave $(n=2)$ sinusoidal curve. It has been assumed that during the buckling process, the track will deform by amplifying the existing misalignment (fig. 1). According to this hypothesis, the generalized coordinates of the system will be the length of the misalignment $L$ and the amplitude of the lateral deflection $y_{0}$. A compressive point load $P$ will be applied in both ends of the misalignment. 
The ballast resistance $\varphi$ opposed to the lateral displacement of the track will be taken into account as a distributed load. For simplicity, ballast resistance is held constant, assuming the limit resistance as a representative value of the total lateral resistance (fig. 2) exerted by ballast. This lateral resistance is provided through the contact between ballast and sleepers at the sleeper base, its sides and its comprised end. The components of the lateral resistance have different characteristics and contribute differently to the total resistance. The resistance provided by the base contact $\varphi_{\mathrm{b}}$ is a friction force, which depends on the vertical load $N_{\mathrm{x}}(\mathrm{x})$ per track unit of length and the friction coefficient $\mu$ between sleeper and ballast [13]:

$\varphi_{b}(x)=f\left(N_{x}(x), \mu\right)$

This vertical load $N_{\mathrm{x}}(\mathrm{x})$ is due to the weight of the track itself, but also to vertical point loads $Q$ originated by passing trains. The spatial distribution and magnitude of these point loads depend on the type of traffic considered in the analysis (fig. 3). The vertical load transmitted to the ballast per unit of length has been calculated using the Zimmerman - method [10]. In the zones adjoining to the point of application of the load, the track is slightly lifted up, losing the contact between sleeper base and ballast. For simplicity, this loss of contact will not be taken into account in the analysis.

The effect of the vertical loads generated by traffic on the value of the base lateral resistance has been obtained by applying the Mohr - Coulomb failure criterion:

$\varphi_{b}^{*}(x)=\varphi_{b}+N_{x}^{Q}(x) \cdot \tan \delta$

where $\varphi_{\mathrm{b}}{ }^{*}(\mathrm{x})$ is the value of the amplified resistance, $N_{x}^{Q}$ is the vertical load per meter of track due to traffic loads $Q, \varphi_{\mathrm{b}}$ the base resistance value for unloaded track and $\delta$ an interface friction angle for the roughened sleeper base, that depends on the material of 
the sleeper. Taking into account that these point loads $Q$ are not static but dynamic, they have been amplified using the Eisenmann formula [14]. The value of the base resistance for an unloaded track can be obtained from the results of Single Tie Push Tests (STPT).

The resistance developed in the comprised end of the sleeper against the ballast $\varphi_{\mathrm{e}}$, is a passive earth pressure [15]

$\varphi_{e}=\frac{1}{2} \cdot\left(K_{P} \cdot \gamma^{\prime}+2 \cdot c^{\prime} \cdot \sqrt{K_{P}}\right) \cdot h^{2} \cdot b$

where $K_{\mathrm{p}}$ is the passive earth pressure coefficient, $\gamma^{\prime}$ the specific weight of ballast, $c^{\prime}$ the cohesion of ballast, $h$ the ballast height over the sleeper base or sleeper ballast coverage, and $b$ the sleeper width.

The resistance provided by the lateral sides of the sleeper $\varphi_{\mathrm{c}}$ is a friction load dependent on the normal pressure exerted by ballast on those sides. If longitudinal displacements of the track are considered negligible, this pressure is equal to the earth pressure at rest [15]:

$\varphi_{c}=2 \cdot N \cdot \mu=2 \cdot\left(\frac{1}{2} \cdot K_{0} \cdot h^{2} \cdot \gamma^{\prime} \cdot a\right) \cdot \tan \delta$

where $N$ is the resultant of the earth pressure at rest on the lateral sides of the sleeper, $K_{\mathrm{o}}$ is the ratio of the horizontal to vertical effective stress in the crib [8], $a$ is the length of the sleeper and $\mu$ is the friction coefficient between ballast and sleeper.

Both resultant of the earth pressure at rest and resultant of the passive earth pressure depend on the dimensions of the sleeper and the geotechnical characteristics of ballast.

The described model also considers the effect of maintenance operations on the ballast resistance. The pass of running vehicles tends to increase the lateral resistance provided by ballast, as ballast gets more and more consolidated. However, the value of the ballast 
lateral resistance is affected by the track maintenance operations that involve ballast, such as tamping or lining [16]. These operations can result in a 50\% to $70 \%$ loss of the lateral resistance existing in a consolidated track (fig. 4). This reduction of the lateral resistance causes a high decrease of the track global rigidity, and buckling may occur. In order to raise the safety of the track against instability, operations like the ones mentioned above are usually combined with dynamic stabilization. Dynamic stabilization of the track may restore up to $80 \%$ of the resistance lost due to tamping operations [16].

It has to be said that passing trains also generate lateral loads on the track, which could trigger the buckling process. However, studies have shown that in straight tracks, these loads have minor effects on the phenomenon [17].

\subsection{Model Resolution}

The total potential $V$ of the comprised track is the sum of the deformation energy associated to bending of the rails, the energy associated to the lateral resistance of ballast, and the work of the external forces. Considering all the hypotheses mentioned above, the total potential of the track system can be formulated as

$V\left(y_{0}, L, F\right)=U_{\text {bending }}+U_{\text {ballast }}-W_{P}$

where

$$
\begin{aligned}
& U_{\text {bending }}=\frac{1}{2} \int_{0}^{L} E I_{h} y^{\prime \prime 2} d x \\
& U_{\text {ballast }}=\int_{0}^{L} \varphi \cdot y d x \\
& W_{p}=\frac{1}{2} \int_{0}^{L} P\left[\left(y^{\prime}+f^{\prime}\right)^{2}-f^{\prime 2}\right] d x
\end{aligned}
$$


The function $y$ is the lateral deflection of the CWR track due to buckling, which according to the described hypotheses will be a sinusoidal function. As can be seen, the potential is a function of the unknowns of the problem, namely the generalized coordinates $L$ and $y_{\mathrm{o}}$, and the external applied compressive force $P$.

The energy of the resistance forces exerted by ballast has been decomposed in its three components: base, crib and end resistance, each of them dependent on different factors $U_{\text {ballast }}=\int_{0}^{L}\left(\varphi_{b}^{*}+\varphi_{l}+\varphi_{e}\right) \cdot y d x=\int_{0}^{L} \varphi_{b}^{*} \cdot y d x+\int_{0}^{L} \varphi_{l} \cdot y d x+\int_{0}^{L} \varphi_{e} \cdot y d x$

The buckling load can be determined by applying the equations that characterize a neutral equilibrium state, which are:

Equilibrium equation $1: \frac{\partial V\left(y_{0}, L, F\right)}{\partial y_{0}}=0$

Equilibrium equation 2: $\frac{\partial V\left(y_{0}, L, F\right)}{\partial L}=0$

Condition for neutral equilibrium: $\left|\begin{array}{ll}\frac{\partial^{2} V\left(y_{0}, L, F\right)}{\partial y_{0}{ }^{2}} & \frac{\partial^{2} V\left(y_{0}, L, F\right)}{\partial y_{0} \partial L} \\ \frac{\partial^{2} V\left(y_{0}, L, F\right)}{\partial L \partial y_{0}} & \frac{\partial^{2} V\left(y_{0}, L, F\right)}{\partial L^{2}}\end{array}\right|=0$

The resolution of this equation system provides not only the buckling load of the comprised track, but also the amplitude of the deformation when buckling begins to develop and the length of the misalignment for which the buckling load is lower and therefore critical for the analyzed track. That means that if the track has misalignments with a different length than the one calculated with the model, the buckling load will be higher.

\section{Parametric study}

A parametric study has been performed using the model described above in order to evaluate the effects of the considered parameters on the track buckling load. The study focuses on the effect of the sleeper type and material, rail type, amplitude and form of 
the initial misalignment, ballast height over the base of the sleepers (from now on sleeper ballast coverage) and lateral ballast resistance affected both by maintenance operations and by passing traffic.

Fixed values for some parameters have been considered in the analysis (table 1). The considered spacing between centers of mass of two consecutive sleepers is $0.6 \mathrm{~m}$. Two different rail types have been considered, namely UIC54 and UIC60. The sleeper types considered are conventional wooden sleepers, single block concrete sleepers, and twin block concrete sleepers. Depending on the sleeper material, for concrete sleepers the friction coefficient is $\mu=0.86$, and for wooden sleepers $\mu=1.2$. The reference values for total lateral ballast resistance from which to obtain the base resistance for an unloaded track are shown in the table 2.

The parametric study has been performed as follows. At first, the CWR track buckling load has been calculated using the proposed analytical model for each possible combination of the considered parameters. After that, a statistical treatment of the 720 obtained results has been done using Statgraphics Centurion $X V{ }^{\circledR}$. Two different statistical analyses have been done, depending on if the track is loaded or not. It is expectable that a loaded track will show a higher buckling load than an unloaded track. However, one of the objectives of this survey is to quantify this safety increase. Different levels for each factor have been evaluated (table 3). Each level is characterized by the mean buckling load obtained from all the results in which the parameter has been held at this level.

The global effect $X_{\mathrm{G}, \mathrm{i}}$ of a single parameter $i$ is determined as the difference between the mean buckling loads obtained for the different levels of the parameter (fig. 5).

$$
X_{G, i}=\operatorname{máx}\left\{\bar{P}_{i, j}-\bar{P}_{i, k}\right\}
$$


If more than two different levels of a parameter are evaluated, as in the case of the sleeper type, the global effect will be considered as the maximal difference between the average buckling loads of the evaluated levels.

Once the global effect of each parameter is known, the influence $I_{\mathrm{i}}$ of the parameter $i$ is calculated by dividing its respective effect and the sum of all effects.

$$
I_{i}=X_{G, i} / \sum_{k} X_{G, k}
$$

\section{Results and discussion}

Fig. 6 shows the mean buckling load obtained for each type of sleeper considered in the analysis, for a loaded and for an unloaded track. As can be seen, the buckling load for a track with wooden sleepers is approximately $20 \%$ lower than the buckling load for a track with single block concrete sleepers. For concrete bi-block sleepers the buckling load is $5 \%$ higher than for conventional concrete sleepers. This is due to the fact that with bi-block sleepers, the passive earth pressure is developed by two ends instead of only one. However, the crib resistance of ballast is lower, due to the reduced lateral area of the bi-block sleepers. Both of the mentioned effects compensate and because of that, the total safety increase associated to bi-block sleepers is so reduced.

Fig. 7 shows the effect of the considered rail type on the value of the track buckling load. It can be seen that for UIC60 rails, the buckling load for the track is $11 \%$ higher than for UIC54 rails. This is a direct consequence of the major inertia in the horizontal plane of the UIC60 rails and its major contribution to the global rigidity of the track.

As can be seen in fig. 8 , a recently tamped track shows a mean buckling load $20 \%$ lower than the buckling load for a consolidated track because of the reduction of the lateral resistance of ballast. With the dynamic stabilization, the safety loss associated to 
tamping can be reduced by $50 \%$. This shows the importance of combining maintenance operations like tamping with dynamic stabilization, in order to increase the safety of the track against buckling.

Fig. 9 shows the mean buckling load of a CWR track when the ballast is flushed to the upper sleeper surface, and when the ballast height is $5 \mathrm{~cm}$ under the upper sleeper surface. Analytical results show that, in the considered range of variation, the influence of the ballast height on the buckling load of the track is negligible.

Calculations have been made considering an initial misalignment with a half wave and with a full wave sinusoidal curve form. Fig. 10 displays the mean buckling load for both forms of the initial misalignment. It can be seen that for an unloaded track, the form of the initial defect has no relevant influence on the phenomenon.

Two different amplitudes have been also considered for the initial misalignment, namely a $2.5 \mathrm{~cm}$ and a $5 \mathrm{~cm}$ amplitude. For an unloaded track, the mean buckling load for $2.5 \mathrm{~cm}$ amplitude is about $40 \%$ higher than for $5 \mathrm{~cm}$ amplitude. As can be seen in fig. 11, this difference is higher when the track is loaded. From these results it can be concluded that maintenance operations that correct track geometrical misalignments are necessary to guarantee the stability of a comprised CWR track.

In figs. 6 to 11 the mean buckling loads are shown for both loaded and unloaded track. On average, the buckling loads for loaded tracks are over $200 \%$ higher than for unloaded tracks. This is so because the applied vertical loads tend to increase the lateral resistance exerted by ballast, leading to a higher global rigidity of the buckling system. The increase on the buckling loads depends on the vertical loads applied (the value of the loads and their spatial distribution along the track) and the considered traffic speed. 
According to the results obtained using the analytical model, the relative influence of the factors on the buckling phenomenon has been calculated as explained in the previous section. Table 4 shows the considered parameters and their quantitative influence on the CWR track buckling process.

It can be concluded that the most important factors for an unloaded track are the type of sleeper, the track maintenance operations that involve ballast, and the amplitude of the misalignment. For a loaded track, however, only the parameters that define the misalignment (form and amplitude) seem to be important. This can be explained because the rest of the parameters mostly influence the crib and end resistance exerted by ballast. When a significant vertical load is applied on the track, the increase of its global rigidity is mainly due to the base resistance of ballast. Therefore, variations of the other factors have minor effects on the global rigidity of a loaded track.

\section{Conclusions}

In this survey, an analytical model has been developed in order to quantify the influence of different factors on the CWR track buckling phenomenon. The main contributions of this model are that it allows the calculation of the buckling load of a CWR track, considering the effect of track vertical loading and the variation of the sleeper ballast coverage on the total ballast lateral resistance. It also provides a buckling load non dependent on an initially supposed length of the existing track misalignment. However, the formulation still presents some limitations: lateral resistance provided by ballast has been held constant and the effect of the lift-up wave of the loaded track has been neglected. These parameters should be considered more accurately in future models. 
The main findings of the parametric study accounting for the rail and sleeper type, the amplitude and form of the initial misalignment, loss of ballast height and lateral ballast resistance are:

1) Results show that the most important factors involved in the buckling process are the sleeper type, the characteristics of the initial misalignment and the maintenance operations that affect the ballast.

2) The ballast height over the sleeper base, at least in the considered range of variation, seems to have no influence on the track rigidity.

3) The form of the misalignment for unloaded track and the rail type do have an influence on the phenomenon, but almost negligible compared with the first mentioned parameters.

4) Buckling is much more likely to develop when the track is unloaded than when trains are passing, because of the increase of ballast resistance due to vertical loading. For a loaded track, the buckling load is over $200 \%$ higher than for an unloaded track.

5) Dynamic stabilization is very advisable in order to lessen the loss of global track rigidity due to tamping and other maintenance operations, which highly reduce the ballast resistance against lateral displacement of the CWR track.

\section{Fundings}

Project funded by the Spanish Ministry of Education

\section{References}


[1] L. Sabuco, Capsuled History of Railwelding. http://www.hollandco.com/railwelding-services/images/History\%20of\%20Railwelding(PDF).pdf （accessed: January 2013)

[2] Volpe National Transportation Systems Center, Track Buckling Research. www.volpe.dot.gov/coi/pis/work/archive/buckling.html (accessed: January 2013)

[3] M.A. Van, Buckling Analysis of Continuous Welded Rail Track, HERON 41 (1996).

[4] N.-H. Lim, S.-Y. Han, T.-H. Han, and Y.-J. Kang, Parametric Study on Stability of Continuous Welded Rail Track - Ballast Resistance and Track Irregularity, International Journal of Steel Structures 8 (2008), pp. 171-181.

[5] A.D. Kerr, Analysis of Thermal Track Buckling in the Lateral Plane, Acta Mech. 30 (1978), pp. 17 - 50.

[6] G.T. Grissom, and A.D. Kerr, Analysis of lateral track buckling using new frame-type equations, International Journal of Mechanical Sciences 48 (2006), pp. 21-32.

[7] G. Samavedam, F. Blader, and D. Thomson, Safety of High Speed Ground Transportation Systems Track-Lateral Shift: Fundamentals and State-of-the-Art, U.S. Department of Transportation, 1996.

[8] M. Kaya, A Study on the Stress-Strain Behavior of Railroad Ballast Materials by Use of Parallel Gradation Technique. PhD, The Middle East Technical University, 2004. 
[9] C. Esveld, Modern Railway Track, 2nd ed. MRT-Productions, 2001.

[10] A. López Pita, Infraestructuras Ferroviarias, Ediciones UPC, 2006.

[11] ITEA (Instituto Técnico de la Estructura en Acero) .ESDEP, European Steel Design Education Programme.

[12] J.A. Zakeri, Lateral Resistance of Railway Track, in Reliability and Safety in Railway, 2012, pp. 357 - 374.

[13] A. Kish, On the Fundamentals of Track Lateral Resistance, AREMA 2011 Annual Conference, Minneapolis, 2011.

[14] V.A. Profillidis, Railway Engineering, 2nd ed., Ashgate, 2000.

[15] L.M. Le Pen, and W. Powrie, Contribution of base, crib, and shoulder ballast to the lateral sliding resistance of railway track: a geotechnical perspective, Proceedings of the Institution of Mechanical Engineers Part F-Journal of Rail and Rapid Transit 225 (2011), pp. 113-128.

[16] T. Sussmann, A. Kish, and M. Trosino, Investigation of the Influence of Track Maintenance of Lateral Resistance of Concrete Tie Track, Transportation Research Board Conference, 2003.

[17] Gallego, J. and Gómez-Rey, D. A Finite Element Solution for the Lateral Track Buckling Problem. http://www.uic.org/cdrom/2001/wcrr2001/pdf/sessions/ 2_3_4/441 .pdf (accessed July 2012)

\section{Captions}


Fig 1. Track model with (a) full wave sinusoidal initial misalignment, (b) half wave sinusoidal initial misalignment.

Fig 2. Consideration of ballast total lateral resistance in the model.

Fig 3. Traffic loads configuration.

Fig 4. Influence of maintenance operations in total ballast lateral resistance.

Fig 5. Calculation of the global effect of each parameter.

Fig 6. Effect of the sleeper type on the buckling load.

Fig 7. Effect of the rail type on the buckling load.

Fig 8. Effect of maintenance operations on the buckling load.

Fig 9. Effect of sleeper ballast coverage on the buckling load.

Fig 10. Effect of the misalignment form on the buckling load.

Fig 11. Effect of the misalignment amplitude on the buckling load. 